\title{
RESEARCH
}

Open Access

\section{Quantitative sequencing clarifies the role of disruptor taxa, oral microbiota, and strict anaerobes in the human small-intestine microbiome}

Jacob T. Barlow ${ }^{1 \dagger}$, Gabriela Leite ${ }^{2 \dagger}$, Anna E. Romano ${ }^{3}$, Rashin Sedighi ${ }^{2}$, Christine Chang ${ }^{2}$, Shreya Celly ${ }^{2}$, Ali Rezaie ${ }^{2,4}$, Ruchi Mathur ${ }^{2,5}$, Mark Pimentel ${ }^{2,4}$ and Rustem F. Ismagilov ${ }^{1,3^{*}}$ (D)

\begin{abstract}
Background: Upper gastrointestinal (Gl) disorders and abdominal pain afflict between 12 and 30\% of the worldwide population and research suggests these conditions are linked to the gut microbiome. Although largeintestine microbiota have been linked to several Gl diseases, the microbiota of the human small intestine and its relation to human disease has been understudied. The small intestine is the major site for immune surveillance in the gut, and compared with the large intestine, it has greater than 100 times the surface area and a thinner and more permeable mucus layer.
\end{abstract}

Results: Using quantitative sequencing, we evaluated total and taxon-specific absolute microbial loads from 250 duodenal-aspirate samples and 21 paired duodenum-saliva samples from participants in the REIMAGINE study. Logtransformed total microbial loads spanned 5 logs and were normally distributed. Paired saliva-duodenum samples suggested potential transmission of oral microbes to the duodenum, including organisms from the HACEK group. Several taxa, including Klebsiella, Escherichia, Enterococcus, and Clostridium, seemed to displace strict anaerobes common in the duodenum, so we refer to these taxa as disruptors. Disruptor taxa were enriched in samples with high total microbial loads and in individuals with small intestinal bacterial overgrowth (SIBO). Absolute loads of disruptors were associated with more severe Gl symptoms, highlighting the value of absolute taxon quantification when studying small-intestine health and function.

Conclusion: This study provides the largest dataset of the absolute abundance of microbiota from the human duodenum to date. The results reveal a clear relationship between the oral microbiota and the duodenal microbiota and suggest an association between the absolute abundance of disruptor taxa, SIBO, and the prevalence of severe Gl symptoms.

Keywords: Duodenum, Saliva, HACEK, Human small intestinal microbiome, IBS, SIBO, Enterobacteriaceae, Lactobacillus, Constipation, Bloating

\footnotetext{
* Correspondence: rustem.admin@caltech.edu

${ }^{\dagger}$ Jacob T. Barlow and Gabriela Leite contributed equally to this work.

'Division of Biology and Biological Engineering, California Institute of

Technology, 1200 E. California Blvd, Pasadena, CA 91125, USA

${ }^{3}$ Division of Chemistry and Chemical Engineering, California Institute of

Technology, 1200 E. California Blvd, Pasadena, CA 91125, USA

Full list of author information is available at the end of the article
} 


\section{Background}

Hundreds of studies have linked the human microbiome to specific diseases. In metabolic diseases or gastrointestinal (GI) disorders (e.g., irritable bowel syndrome [IBS], Crohn's disease, malabsorption) that can cause GI symptoms, such as pain, bloating, and diarrhea, the small intestine instead of the colon may be the primary site of microbial interactions related to disease. Studies have focused on stool primarily for its ease of access and the fact that it has the highest density of microbes out of any human sample type [1]. The stool microbiome has been shown to be a good proxy for the large-intestine microbiome, but is known to differ substantially from the small-intestine microbiome [2, 3]. Compared with the large intestine, the small intestine has several physiological differences that indicate its potential relevance for microbial interactions. The surface area of the small intestine is greater than 100 times that of the large intestine, underlining its role in nutrient absorption. Additionally, the mucus layer of the small intestine is much thinner and more diffuse [4], potentially allowing closer interactions between microbes and the host. Finally, the small intestine is the main site for intestinal immune surveillance by lamina propria dendritic cells [5] and Peyer's patches [6], contributing to the body's response to both commensal and pathogenic microbes.

Although mouse studies have been an insightful proxy for understanding the large-intestine microbiome of humans, the coprophagic behavior of mice [7] and many other animal models results in a substantially different small-intestine microbiome compared with humans [8]. For example, the total microbial load of the human small intestine is generally thought to be low, around $10^{2}-10^{6}$ $\mathrm{CFU} / \mathrm{mL}$ [1], whereas microbial loads in laboratory mice are nearly $10^{9} \mathrm{CFU} / \mathrm{mL}[8,9]$. In humans, culturable levels above $10^{3}-10^{5} \mathrm{CFU} / \mathrm{mL}$ from duodenal aspirates are used as the clinical determination of small intestinal bacterial overgrowth (SIBO) [10]. SIBO has been shown to correlate with IBS and GI symptoms such as bloating, constipation, and diarrhea $[11,12]$. Physiologically, SIBO has also been linked to slow intestinal transit [13], higher body mass index (BMI) [14], and reduced stomach-acid levels [15]. Standard-of-care treatments for SIBO often include antibiotics and diets designed to reduce the amount of rapidly fermentable products in the small intestine [16]. However, reoccurrence of symptoms after antibiotics is common and adherence to strict diets is often difficult for patients [17]. Only recently has a connection between the relative abundance of specific microbial taxa, generally from the Enterobacteriaceae family, and SIBO begun to be uncovered [18].

The difficult nature of sampling most of the gastrointestinal tract has resulted in a limited number of studies analyzing the microbial composition of the human small intestine. Several studies have relied on sampling from ileostomy bags $[19,20]$, but such sampling will not be fully representative of the small-intestine microbiome [21]. More recent studies sample directly from the intact small intestine through an endoscopic procedure and have begun to unravel unique relationships between small-intestine microbes and disease $[18,22-25]$. An added challenge when quantifying individual microbial taxa from samples of low total microbial biomass is that it can be difficult to distinguish true small-intestine microbes from contamination (e.g., from the oral cavity while sampling or from reagents during sample processing). Additionally, the wide range of total microbial loads in the small intestine across individuals highlights the value of using absolute rather than relative microbial loads when investigating potential associations between small-intestine microbes and physiological factors [9, 26, 27].

In this study, we selected a cohort of 250 individuals from the REIMAGINE study [3] to assess the absolute microbial loads in the human duodenum and their potential relationship with factors related to health and disease. We also surveyed the oral microbiome in a subset of 21 individuals from this cohort to understand the relationship between microbial taxa at these two body sites. We utilized our recently developed digital PCR anchored 16S rRNA gene amplicon sequencing method to provide absolute taxon abundances and filter out contaminants in samples with low microbial abundance [9]. We also used our optimized sample-collection procedure with a custom double lumen sterile closed catheter system and optimized processing steps to minimize oral, gastric and dead microbial contamination [28]. We hypothesized that by capturing the absolute microbial abundances of the human duodenal and oral microbiome we would be able to better understand the makeup of the human duodenal microbiome, improve the understanding of the underlying community structure of SIBO, and determine how microbial load and composition correlate with upper GI symptoms.

\section{Results}

We studied the microbiome of the duodenum and its potential relationship with health and disease in a cohort of 250 patients enrolled in the REIMAGINE study at Cedars-Sinai Medical Center. All patients undergoing esophagogastroduodenoscopy (EGD) without colonoscopy preparation as standard of care were eligible to enroll, resulting in patients with a wide range of GI conditions. We grouped the reason for endoscopy into 11 broad categories (Table S1). The most common (45\% of the patient population) reasons for endoscopy were to rule out cancer/polyps and GERD/dyspepsia workup. No 
healthy controls are currently approved to be included in the study due to the risks associated with the EGD procedure. Summary statistics for patient demographic data and selected metadata categories from the enrollment questionnaire are included in Table S1.

\section{Total microbial load of the duodenum across patients with Gl symptoms is log-normally distributed}

A digital PCR-based determination of total microbial load $[9,29]$ from 250 human duodenal aspirates revealed samples that spanned loads from our detection limit of $\sim 5 \times 10^{3}$ rRNA gene copies/mL up to nearly $10^{9}$ copies $/ \mathrm{mL}$. The overall distribution of total loads was lognormal with mean $=6.13 \log _{10}$ copies $/ \mathrm{mL}$ and standard deviation $=1.12 \quad \log _{10} \quad$ copies $/ \mathrm{mL}$ (Fig. 1A, B). A quantile-quantile (QQ) plot was constructed to compare the sample distribution to a log-normal distribution (Fig. 1B). Data from our samples aligning with the $y=x$ line on a QQ-plot indicate a high similarity between the sample distribution and a theoretical log-normal distribution [32]. Neither age nor gender significantly correlated with total microbial load (Fig. S1). Total microbial load also did not correlate with patient reported intake of probiotics supplements or yogurts, smoking, or usage of proton pump inhibitors (Fig. S2, Table S2). Current antibiotic usage appeared to lower the average total microbial load, but antibiotic usage in the previous 6 months had no impact (Fig. S2, Table S2).

Digital PCR anchored 16S rRNA gene amplicon sequencing [9] (hereafter quantitative sequencing) provided absolute taxon abundances in each sample and a statistical framework for differentiation between real and contaminant taxa (Methods). We first compared the culture counts from aerobic (MacConkey agar) and anaerobic (blood agar) plates to the total load of microbes expected to grow on these plates (Fig. S3). For aerobic plating, we observed a bimodal distribution of combined Escherichia-Shigella, Enterobacteriaceae, Enterococcus, and Aeromonas bacterial load from quantitative sequencing and culture and a high correlation between the two measurements (Spearman, 0.61, $P<0.001, N=244$ ). For anaerobic plating, we observed lower concordance (Spearman, 0.35, $P<0.001, N=244$ ) between quantitative sequencing and culture. This discrepancy could reflect the difficulty in culturing many intestinal microbes [33], especially anaerobes that are initially collected and processed in aerobic environments.

Next, we analyzed the log-transformed absoluteabundance distributions for the most prevalent genera in our dataset (Fig. 1C). We define prevalence as a taxon's frequency of occurrence in our dataset. Streptococcus was present in all 250 samples and followed an approximately log-normal distribution with a mean load that

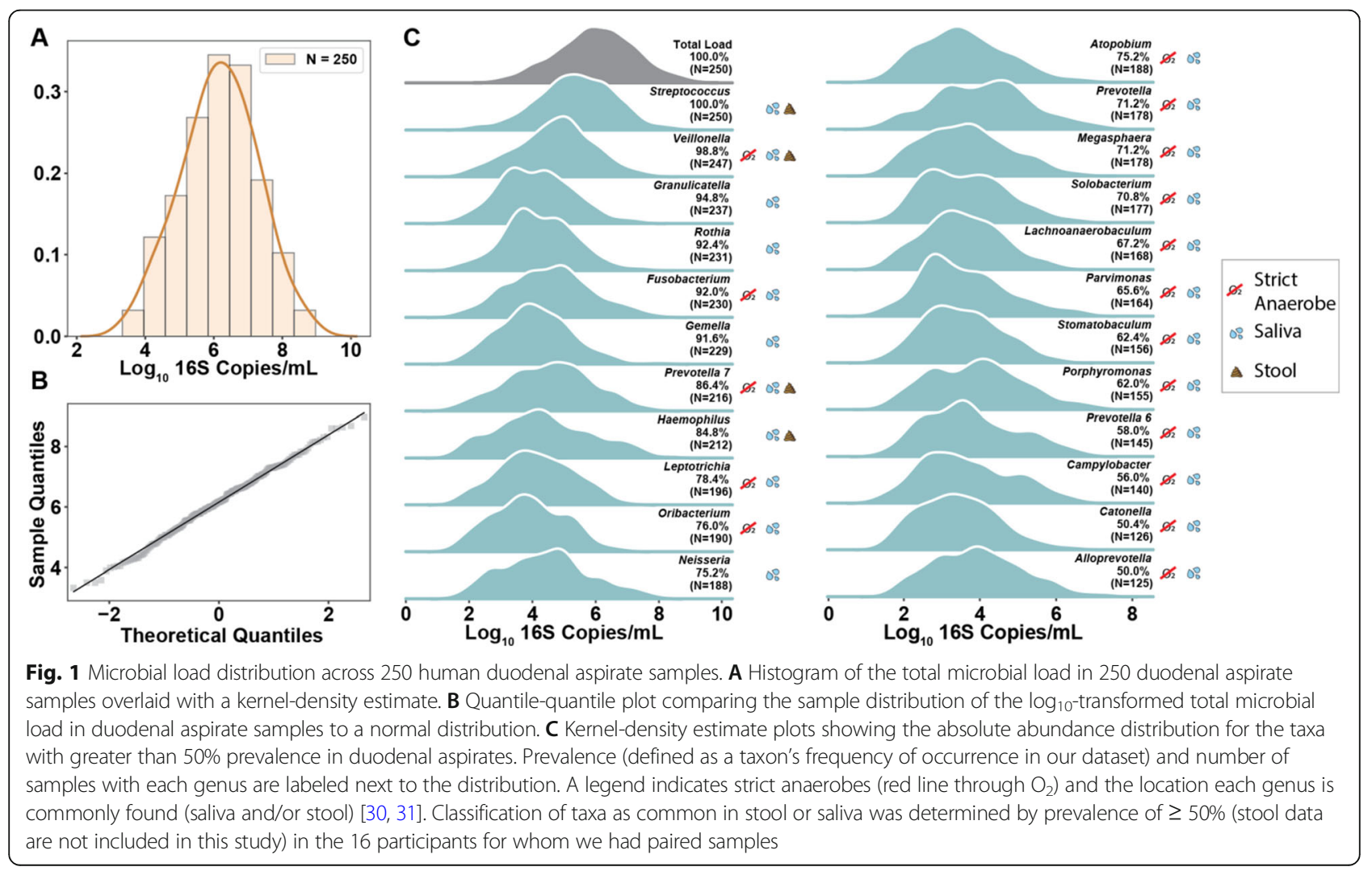


was half an order of magnitude below that of the mean total microbial load and an equal standard deviation. Other genera showed wide-ranging distributions that deviated from normality. For example, Porphyromonas appears bimodal with two local maxima whereas Haemophilus exhibits a long tail towards higher microbial loads. The 23 most prevalent genera in this study are also commonly found in the oral microbiota [30]. A subset of these genera (Streptococcus, Veillonella, Prevotella 7, Haemophilus) are also commonly found in stool samples, indicating possible survival of these genera throughout the entire GI tract [31]. The majority of prevalent genera are either strict or facultative anaerobes, indicating that parts of the duodenal environment are likely anoxic in this patient population.

Direct transmission of microbes from saliva to duodenum To investigate whether many of the taxa found in the duodenum originated from the oral cavity we analyzed a subset of 21 patients for whom we had paired saliva and duodenum samples that were collected during the same hospital visit. Digital PCR revealed that the total microbial load in saliva was roughly 2.5 orders of magnitude higher than the total load in the duodenum (KruskalWallis, $P<0.001$ ).

Further, the range in saliva total loads was 3 orders of magnitude smaller than the range in total loads of the duodenum samples (Fig. 2A). No significant correlation was observed between the total microbial loads in paired saliva and duodenum samples (Fig. 2B). In this study, all samples were collected with a custom double-sheathed catheter via endoscope (see "Methods" section) that moves beyond the outer sheath before aspirating duodenal fluid. This custom catheter should limit oral microbiota contamination of the duodenum during the procedure. Additionally, the optimized sample-processing protocol (see "Methods" section) should eliminate extracellular DNA from swallowed dead bacteria.

To evaluate the direct transmission of microbes from saliva to duodenum, we compared the shared taxa between paired (same patient) and randomly paired samples from the same dataset. On average, $89 \%$ ( $\pm 6 \%$ S.D.) of the taxa in the duodenum were also found in the paired saliva sample, whereas only $66 \%( \pm 9 \%$ S.D.) were found in the average of all non-paired comparisons (Fig. $2 \mathrm{C}$, Kruskal-Wallis, $P<0.001$ ), suggesting direct transmission of oral taxa to the duodenum. We then looked for genera that were proportionally enriched in either saliva or duodenum samples. Campylobacter was present in $21 / 21$ saliva samples but only $10 / 21$ duodenum samples. The absence of Campylobacter in about half of the paired duodenum samples indicates the oral cavity may be the preferred niche of Campylobacter or that Campylobacter has a high sensitivity to the antibacterial properties of the stomach and small intestine [34] (Fig. 2D). In contrast, an undefined species of Streptococcus was only found in duodenum samples (6/21) (Fig. 2D). A breakdown of the difference between duodenal and saliva abundance of all taxa is provided in Table S3. These differences in the relative abundance of specific taxa of microbes between paired saliva and duodenum samples also provide evidence against oral contamination in the duodenal samples.

\section{Taxa co-correlations reveal disruptor taxa}

We assumed that the taxa with the highest absolute abundance would have the highest potential for impacting the host. Thus, we began by analyzing the relationships between the top 20 most abundant genera. A co-correlation heatmap of these taxa revealed several distinct motifs (Fig. 3A): (1) taxa whose absolute loads had a high correlation with total load, (2) taxa whose absolute loads had a higher cocorrelation with another taxon's absolute load than with total microbial load, (3) taxa with a mutually exclusive relationship with almost all other abundant taxa. Examples of the first motif are in the first column/row of the co-correlation heatmap in Fig. 3A. Correlation with total load was often an indicator of a prevalent taxon because the variance in total microbial load was larger than the variance in relative abundance. When two taxa have a higher co-correlation with each other than with total load (motif 2), it potentially indicates these taxa share preferred environmental factors or directly cooperate. One group of these co-correlating taxa that included several Prevotella species and a species of Porphyromonas matches a known shared metabolic niche in the oral cavity [35, 36] (Table S4).

Several genera stood out as having no significant correlation with almost all other abundant taxa (motif 3): Enterobacteriaceae, Escherichia-Shigella, Clostridium sensu stricto 1, and Lactobacillus (Fig. 3A). For clarification, throughout the manuscript our references to Enterobacteriaceae and Escherichia-Shigella refer to unique sequence variants from the Enterobacteriaceae family, but only Escherichia-Shigella could be classified at the genus level. Based on evidence from a previous study [18] using the REIMAGINE cohort that found Klebsiella in several samples, we decided to measure the abundance of Klebsiella via qPCR in all samples containing a high abundance (at least $10^{5} 16 \mathrm{~S}$ rRNA gene copies/mL) of Enterobacteriaceae. We found that the majority (16/ 22) of the samples with a high abundance of Enterobacteriaceae contained Klebsiella (Fig. S4A). Furthermore, in the samples containing Klebsiella, there was a high correlation (Pearson, 0.88, $P<0.001$ ) between Klebsiella load and Enterobacteriaceae load (Fig. S4B). These taxa appeared to disrupt the commonly observed microbial structure (i.e., the prevalent taxa that generally cocorrelate with one another) of the duodenal microbiome. 


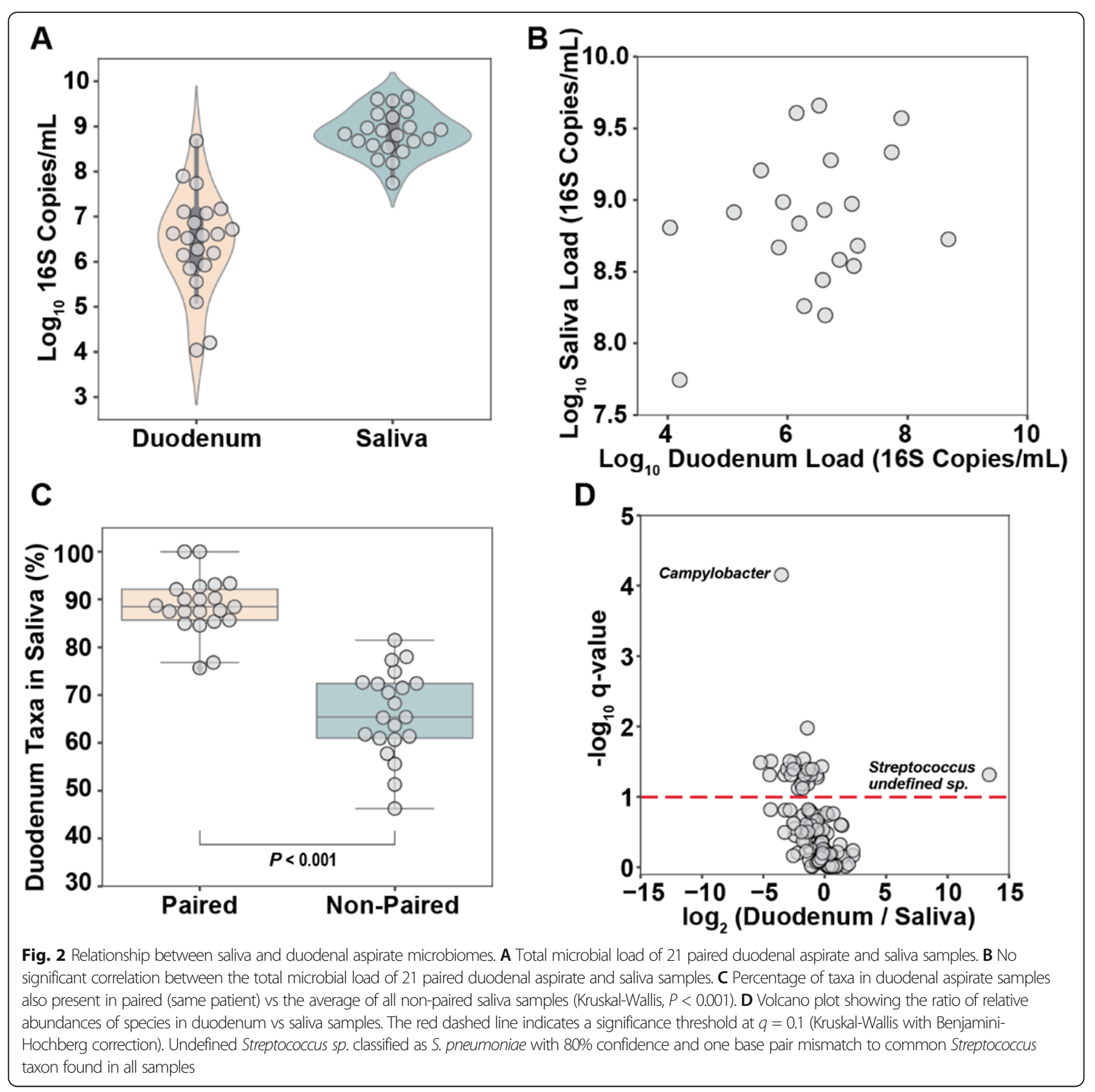

This pattern of mutual exclusivity can be represented algorithmically by sorting all taxa by the difference between their maximum abundance and their mean abundance. Practically, this means that these disruptors are relatively rare (i.e., present in a small fraction of samples), but when they are present they usually dominate, excluding other common taxa. A clustered heatmap of the top 16 taxa as ranked by the difference in their maximum and mean abundances reveals two taxonomic signatures (Fig. 3B). The first signature in the top left of the heatmap contained the mutually-exclusive taxa from the co-correlation heatmap, along with Enterococcus,
Romboutsia, Aeromonas, and Bacteroides. The second signature contained taxa that were generally found in lower abundance, many of which are from the HACEK (Haemophilus, Aggregatibacter, Cardiobacterium, Eikenella, Kingella) group of organisms associated with infective endocarditis [34]. However, the second group also clustered with more common taxa in this dataset, such as Prevotella and Fusobacterium. Thus, we initially labelled all eight of the taxa in the first taxonomic signature as "disruptors" (Fig. 3B, bolded taxa) because their presence appeared to be mutually exclusive with many other common taxa. 


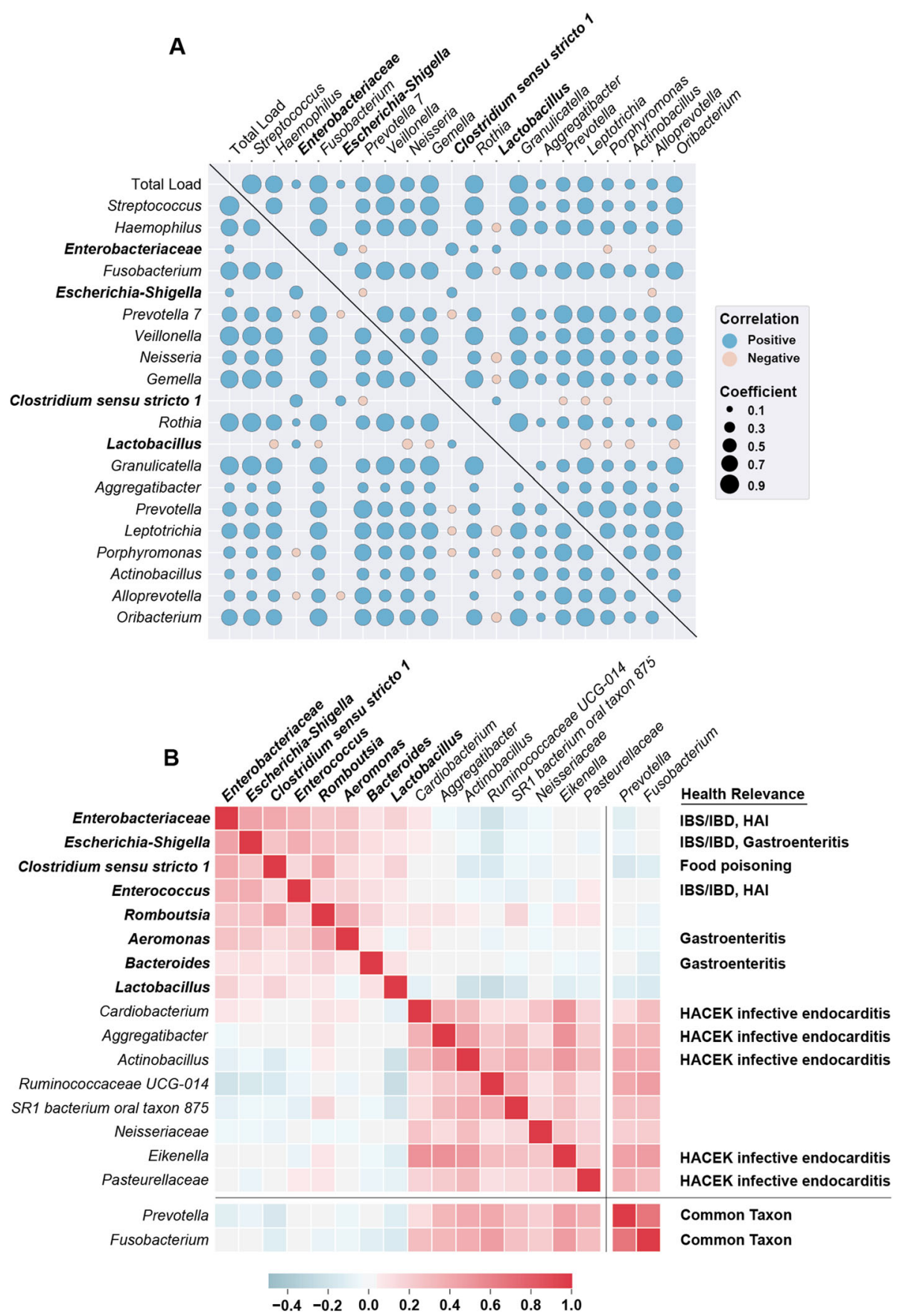

Fig. 3 Co-correlations reveal which taxa co-occur in high abundance and which can be considered disruptor taxa. A Co-correlation matrix of the top 20 most abundant genera and total microbial load. Only significant correlations $(q<0.1$, Benjamini-Hochberg correction) are shown. Color of each marker is determined by the sign of the Spearman's correlation coefficient and size of each marker is determined by the magnitude of the coefficient. Disruptor taxa labels are bolded. B Clustered co-correlation matrix of the top 16 genera ranked by the difference between their maximum abundance and mean abundance. Two common genera in the dataset are shown at the bottom for reference. The color of each square indicates the Spearman correlation coefficient from negative (blue) to positive (red). Disruptor taxa labels are bolded. Taxa with known relevance to human health are indicated. Enterobacteriaceae and Escherichia-Shigella are unique sequence variants from the Enterobacteriaceae family but only Escherichia-Shigella could be classified at the genus level. HAl=hospital acquired infection; IBS, irritable bowel syndrome; IBD, inflammatory bowel disease; HACEK, Haemophilus, Aggregatibacter, Cardiobacterium, Eikenella, Kingella 


\section{Aerobic disruptor taxa displace strict anaerobes and decrease diversity}

After performing the co-correlation analysis, we ran a principal component analysis (PCA) on the absolute taxon abundances to investigate the drivers of variance in the dataset (Fig. 4A). Total loads spanned 5 orders of magnitude, accounting for most of the variance. Total load cleanly separated samples along the PC1 axis. The second most explanatory axis, $\mathrm{PC} 2$, strongly correlated with the Shannon diversity index of samples (Spearman, 0.74, $P<0.001, N=250$ ). Ranked feature loadings for PC2 (Fig. 4B) indicated that many of the disruptor taxa (dark blue) are the main drivers of separation in the positive direction of $\mathrm{PC} 2$ whereas the five taxa driving most of the separation in the negative direction (light blue) of PC2 consisted of four strict anaerobes (Porphyromonas, Leptotrichia, Prevotella, Prevotella 7) and one obligate aerobe (Neisseria). It should be noted that many more taxa were strongly associated with the negative direction of $\mathrm{PC} 2$ than the positive direction. This separation matches well with the mutual exclusivity seen between the disruptor taxa and other organisms in the co-correlation analysis. The two disruptor taxa with the highest loads are aerobic pathogens from the Enterobacteriaceae family and the taxa most associated with the negative direction of PC2 were strict anaerobes, so we next took a closer look at the composition of strict vs facultative anaerobes in each sample. We found a nearly 1:1 correlation between the strict and facultative anaerobe loads across all samples (Fig. 4C). Additionally, the fraction of strict anaerobes in a sample was strongly correlated (Pearson, 0.71, $P<0.001, N=250$ ) with Shannon diversity (Fig. 4D), indicating that the disruptor taxa appear to be mutually exclusive with strict anaerobes and the "bloom" of absolute abundance of disruptors decreases Shannon diversity. Furthermore, in half of the samples containing the two most common disruptor taxa (Enterobacteriaceae and Escherichia-Shigella), the total microbial loads were greater than $10^{7} 16 \mathrm{~S}$ rRNA gene copies $/ \mathrm{mL}$, indicating a clear enrichment of disruptor taxa in samples with higher than average total microbial loads (Fig. 4E). This signature of higher than average total microbial loads and mutual exclusivity with other microbes has been observed in some pathogenic microbial species $[37,38]$.

\section{Absolute load of disruptor taxa correlates with SIBO and Gl symptoms}

To determine whether disruptor taxa are associated with disease or GI symptoms we began by looking at patients with and without SIBO (SIBO classification was made based on aerobic culture results, $\geq 10^{3} \mathrm{CFU} / \mathrm{mL}$ of

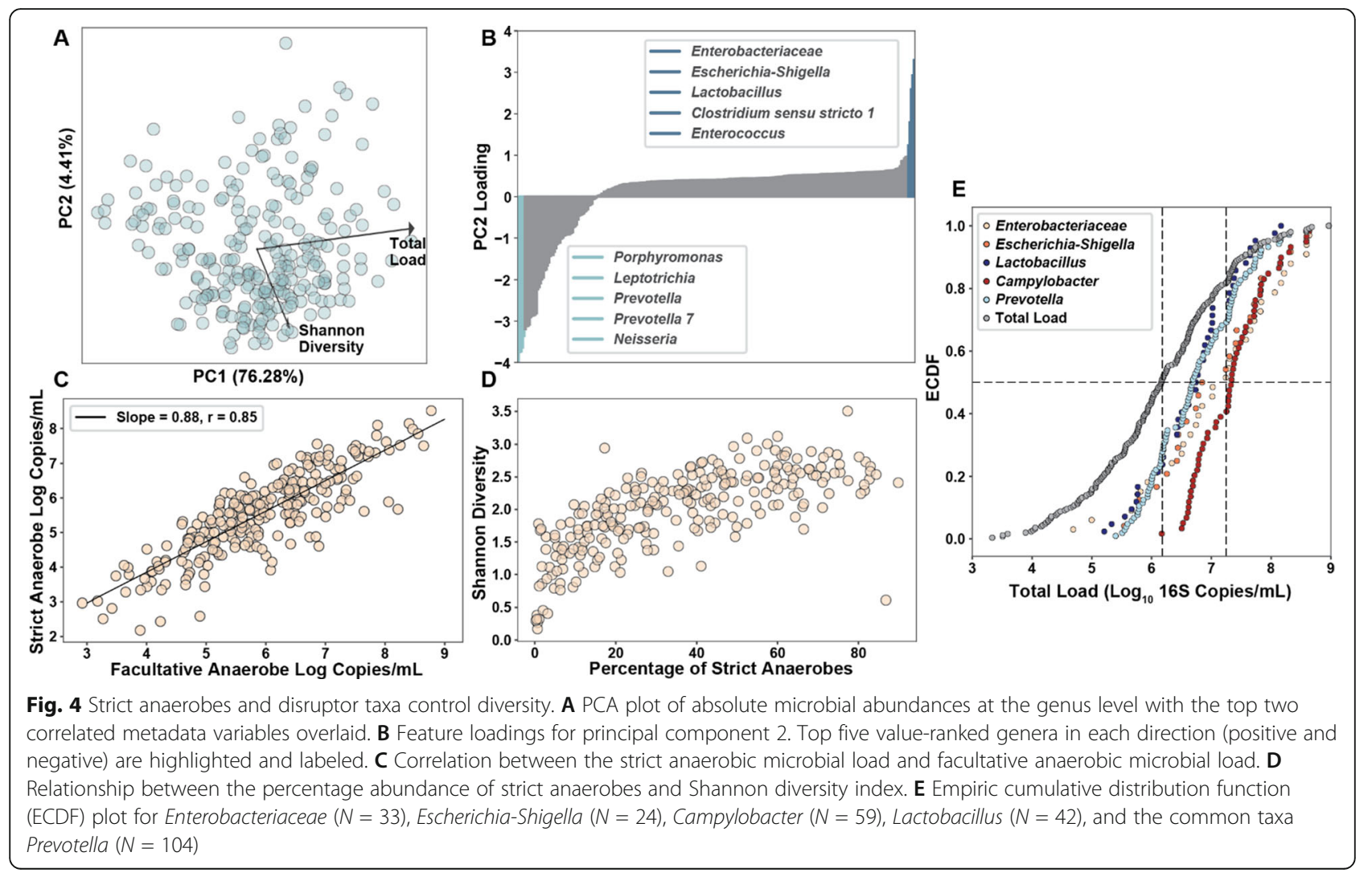


duodenal aspirate [10]). Coloring the PCA plot by SIBO classification indicates a clear enrichment of patients with SIBO in the positive direction of the disruptor taxa axis (Fig. 5A). We observed slightly but not significantly higher total microbial loads in samples from patients with SIBO vs without SIBO (Fig. 5B). However, comparing the absolute abundance of specific taxa between the SIBO and non-SIBO samples by Kruskal-Wallis showed that the three taxa whose abundances differed the most between SIBO and non-SIBO (Enterobacteriaceae,
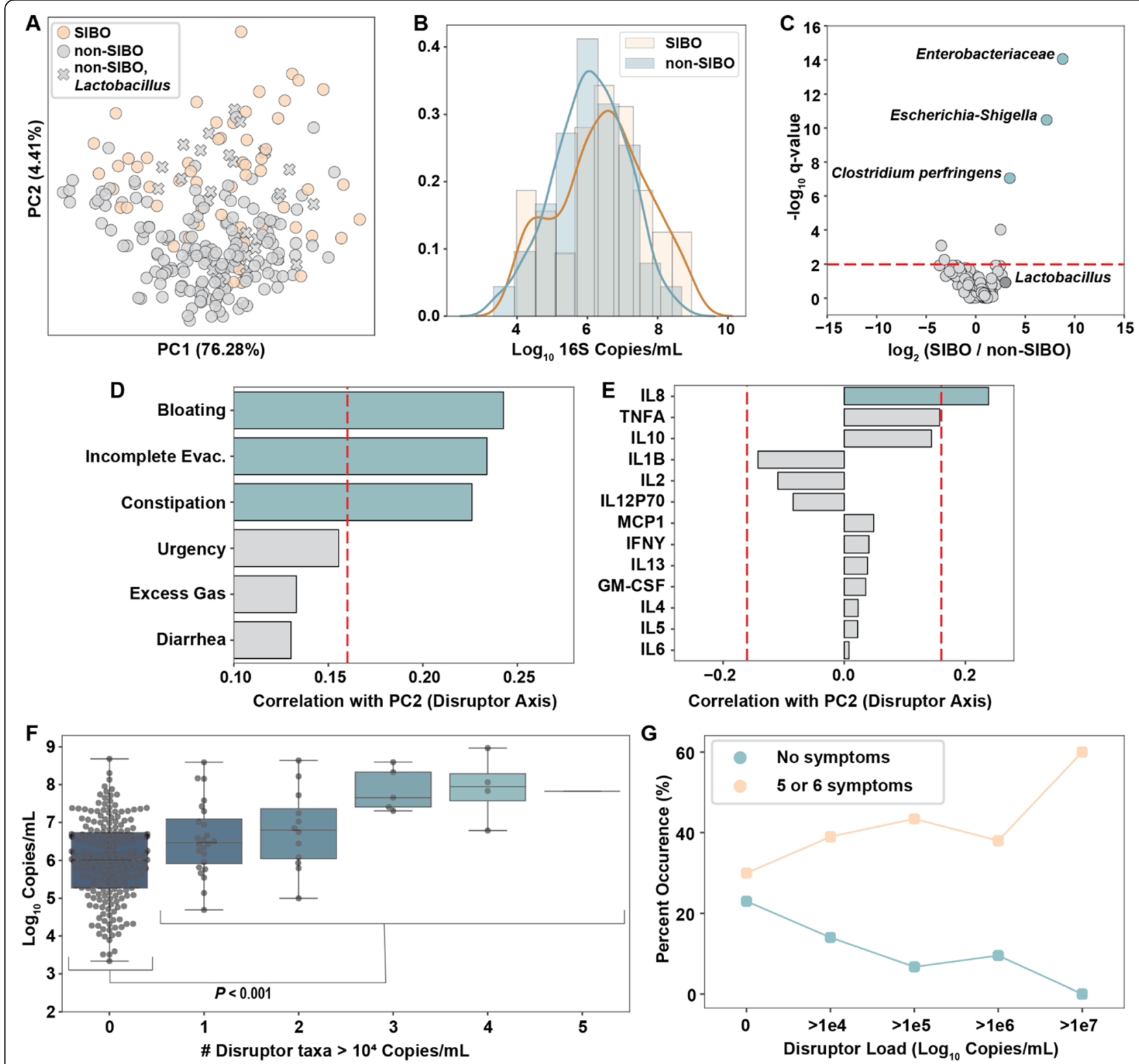

Fig. 5 Disruptor species are dominant in SIBO samples and correlate with Gl symptoms and the inflammatory cytokine IL8. A Principal component analysis (PCA) of absolute microbial abundances at the genus level. Colors indicate non-SIBO (grey) or SIBO (orange) participants as determined by culture. " $X$ " markers indicate samples from non-SIBO participants that contained Lactobacillus. The PC1 axis correlates with total load and the PC2 axis correlates with the abundance of disruptor taxa. B Histogram with overlaid kernel-density estimate of the total microbial loads in samples from SIBO and non-SIBO participants. C Volcano plot indicating the taxa that differed between SIBO and non-SIBO samples. The red dashed line indicates the significance threshold at $q=0.01$. D Correlation between PC2 (disruptor axis) and patient-reported symptom scores (on a 0-100 scale). The red dashed line represents significance threshold at $q=0.05$. E Correlation between PC2 and patient serum cytokine levels. The red dashed lines represent the significance thresholds at $q=0.05$. $\mathbf{F}$ Boxplot indicating increasing average total microbial load with increasing number of disruptor taxa with loads greater than $10^{4} \mathrm{rRNA}$ gene copies/mL (not including Lactobacillus). A significant difference between total load in samples with zero disruptor taxa and total load in samples with at least 1 disruptor taxa was observed $(P<0.001)$. G Percentage of samples from patients with either 0 symptoms or 5-6 symptoms (out of 6 categories) for individuals with varying loads of disruptor taxa (not including Lactobacillus) 
Escherichia-Shigella, and a Clostridium which, based on the V4 region of the 16S rRNA gene, was classified as Clostridium perfringens) were also the three most common disruptor taxa in all samples (Fig. 5C). This enrichment of disruptor taxa, but not total microbial load, in SIBO samples indicates that overgrowth of specific taxa drives the current clinical classification of SIBO. Additionally, using disruptor taxa load as the criterion for SIBO classification agreed well $(80 \%)$ with the classification by the gold-standard method, aerobic aspirate culture (Fig. S5). Lactobacillus abundance was similar in SIBO and non-SIBO samples (Fig. 5C) even though it co-correlated with many of the disruptor taxa (Fig. 3B). Most of the non-SIBO samples that clustered with SIBO samples on the upper part of the PC plot contained Lactobacillus (Fig. 5A). Lactobacillus does not grow on the aerobic (MacConkey agar) plates used for SIBO classification, which could explain why these samples cluster together by sequencing but are not classified as SIBO by culture.

Patient-reported GI symptom scores (on a 0-100 scale) were correlated with the disruptor taxa axis (PC2). Bloating, incomplete evacuation, and constipation had the highest correlation with the disruptor taxa axis, whereas correlations between urgency, excess gas, or diarrhea and the disruptor taxa axis were much weaker (Fig. 5D). There was a weak positive correlation between the disruptor taxa axis and serum interleukin 8 (IL8) levels (Spearman, 0.24, $P<0.001, N=232$ ), indicating a potential neutrophil-related response (Fig. 5E). However, none of the symptoms or cytokines had a significant correlation with the total load axis (PC1). One taxon, which based on the V4 region of the 16S rRNA gene was classified as $C$. perfringens, was the only one that, when present in patients, coincided with a significant increase (Kruskal-Wallis, $P=0.039$ ) in serum IL8 levels (Fig. S6). However, there were only $9 / 250$ samples with $C$. perfringens, limiting our ability to draw conclusions about this relationship. Although the two disruptor taxa with the highest absolute abundance (Enterobacteriaceae and Escherichia-Shigella) were enriched in high total microbial load samples, Lactobacillus did not follow this trend. Lactobacillus was found in samples with total microbial loads that were similar to the total loads of samples containing common taxa like Prevotella (Fig. 4E). Additionally, in patients with high disruptor taxa loads (after excluding Lactobacillus load) the presence of Lactobacillus at greater than $5 \times 10^{4}$ copies/mL negatively correlated with bloating symptoms (Fig. S7). These two facts led us to believe Lactobacillus likely has a more nuanced relationship with the host than the other taxa we classified as disruptors. Thus, we removed Lactobacillus from our list of disruptor taxa in our analyses of the association of disruptors with total load (Fig. 5F) and GI symptoms (Fig. 5G). When multiple disruptor taxa were present, there was a significant increase in total microbial load (Kruskal-Wallis, $P<0.001$; Fig. 5F).

Patient-reported symptom scores are inherently qualitative, so to test whether disruptor taxa loads were correlated with more severe GI symptoms, we turned the 0100 scores into a binary yes/no variable, representing a severe symptom, by drawing a threshold at the median score reported for each symptom (Fig. S8). We then calculated the percentage of patients with zero severe symptoms and the percentage of patients with many severe symptoms (people reporting severe symptoms in 56 of the 6 symptom categories) as a function of disruptor taxa loads (Fig. 5G). We made three observations. First, at higher disruptor loads, patients were more likely to have more severe GI symptoms. Second, none of the patients with disruptor loads greater than $10^{7}$ copies $/ \mathrm{mL}$ $(N=10)$ had zero symptoms whereas $60 \%$ of them had 5 or 6 symptoms. Of the patients without disruptor taxa $(N=153), 23 \%$ had zero symptoms and $30 \%$ had 5 or 6 symptoms. Disruptor loads may also be higher as a function of age, all but one of the individuals with disruptor loads greater than $10^{6}$ copies $/ \mathrm{mL}(N=23)$ were older than 50 (Fig. S9). The absolute and relative abundances of disruptor taxa did not correlate (Fig. S10), preventing the clear connection between abundant symptoms and high absolute loads of disruptor taxa from being observed when analyzing only relative abundances.

\section{Discussion}

In this study, we utilized quantitative sequencing to determine the total and taxon-specific loads from the duodenum of 250 patients undergoing EGD as standard of care. We showed that the total microbial load in the duodenum of these patients spans 5 orders of magnitude and follows a log-normal distribution. Paired salivaduodenum samples revealed that on average $89 \%$ of the taxa in the duodenum were also present in paired saliva samples, suggesting potential transmission of taxa from the oral cavity. Co-correlation analysis of the most abundant taxa revealed a distinct taxonomic motif of "disruptor" taxa that, when present, dominate over other taxa. The most common of these disruptor taxa were aerobic pathogens from the Enterobacteriaceae family and were negatively correlated with the presence of strict anaerobes and diversity. In addition to the apparent community disruption, disruptor taxa were enriched in many patients classified as having SIBO and high loads of disruptors correlated with a high prevalence of severe GI symptoms.

\section{Human vs mouse small-intestine microbiome}

Several findings from this study emphasize how different the small-intestine microbiome is between mice and 
humans. Our previous study revealed that the coprophagic nature of mice resulted in total microbial loads spanning approximately one order of magnitude from $5 \times$ $10^{8}-5 \times 10^{9} 16 \mathrm{~S}$ rRNA gene copies/mL [8] in the small intestine while our human cohort spanned 5 orders of magnitude with a median of $10^{6}$ copies $/ \mathrm{mL}$ (Fig. S11). Additionally, neither the most common disruptor family, Enterobacteriaceae, nor any of the taxa with at least 50\% prevalence in this study were commonly found in our previous study examining microbial loads in the mouse small intestine [8]. Instead, in that study we found that the mouse small intestine was dominated by Lactobacillus and, as a result of coprophagy, several stool microbes [8]. The total microbial load of stool is similar between mice and humans [39] and they both share several common taxonomic groups [40]. These differences should be considered when using mice to model human health or disease impacted by the small intestine.

\section{Value of quantitative analysis}

The nearly 5 orders of magnitude spread in total microbial loads in the duodenum of these patients revealed the value of utilizing an absolute abundance measurement technique when analyzing microbial communities. Analyzing absolute abundances of individual taxa also let us filter out likely contaminants using Poisson loading statistics, which is critical for samples with low microbial abundance, such as those sometimes found in the human small intestine $[41,42]$. The range of total loads in saliva and in stool each appear to be smaller than in the duodenum, closer to two orders of magnitude, which likely relates to differences in residence times, nutrient availability, and host defenses at these two sites compared with the small intestine [39]. Another benefit of using absolute rather than relative abundance measurements is the improved accuracy of correlations between microbes and host phenotype. For example, the 10 patient samples with the greatest disruptor loads had the highest prevalence of severe GI symptoms, but these samples had relative abundances of disruptor taxa that ranged from 8 to $97 \%$. This wide range of relative abundances made samples with high disruptor loads indistinguishable from samples with intermediate disruptor loads when analyzing relative abundances.

\section{Microbial connection between oral cavity and small intestine}

The majority (89\%) of identified microbial taxa in the paired duodenum samples were also present in the paired saliva samples. Our data supports the hypothesis of oral-duodenal transmission of microbes but a larger paired study utilizing shotgun metagenomic sequencing techniques would provide stronger evidence for this claim. Survival of microbes after ingestion is likely dependent on many host factors, including stomach-acid levels, bile secretions, antimicrobial-peptide production, and GI motility. The bimodal taxon abundance distributions (Fig. 1C) observed for some taxa, including Prevotella, may indicate two subsets of patients with distinct stomach and/or duodenal environments that allow for differential abundance of specific taxa. For example, Campylobacter concisus, one of the most common oral Campylobacter species, is known to be sensitive to both stomach and bile acids [34]. Therefore, one could hypothesize that if a patient had low levels of stomach or bile acids some C. concisus may survive ingestion. Low-acid conditions could also allow many other bacteria to survive transit to the duodenum, resulting in higher total microbial loads in the small intestine. We suspect we observed something similar in our samples; the Campylobacter genus was only found in samples with greater than average total microbial loads (Fig. 4E). However, we did not observe a relationship between total microbial loads in the duodenum and the patients' use of proton pump inhibitors (PPI), which are known to reduce acid production. PPI impact on survival of microbes between the oral cavity and duodenum may be dependent on how recently the PPI was taken, however this information was not collected from patients in the REIMAGINE study. A conclusive comparison of the relative importance of various factors affecting bacterial survival in the duodenum would require additional information on small-intestine secretions of bile acids and antimicrobial peptides in these patients.

Several common oral microbes have been implicated in GI diseases when present in stool [30, 43]. A high microbial load in the small intestine could increase the likelihood of these microbes surviving all the way down the GI tract. The shared taxa between the small intestine and oral microbiota in our paired salivaduodenum samples provides evidence that blooms of opportunistic pathogens in the mouth could also lead to colonization in the SI [30]. In this study, only 1 of the 21 paired duodenum-saliva samples contained disruptor taxa in the duodenum, but these taxa were not present in the corresponding saliva sample. Several Enterobacteriaceae species have been identified in oral samples [44] but usually at a low frequency in healthy populations. Many Enterobacteriaceae species are introduced into the gut from contaminated food and water sources [45] which would likely result in only transient oral residence. However, persistent oral Enterobacteriaceae species have been linked to the use of dentures and the presence of periodontal disease [46]. All the taxa we classified as disruptors in this study are more frequently found in stool than in the small intestine or oral cavity [30, 31]. Further studies 
should be performed to determine the source of disruptor taxa in the upper GI tract.

A number of taxonomic groups we identified in the duodenum have members known to be opportunistic pathogens. Beyond disruptor taxa, several taxa from the HACEK group of organisms [47] associated with infective endocarditis were found in high abundance in the duodenum. The route that these and other opportunistic pathogens take to reach the blood stream is not clear but our data show that the HACEK organisms are not limited to the oral cavity. The same traits that allow them to colonize the mouth and heart (biofilm production [48], and general resistance to most host secretions) likely contribute to their ability to survive in the small intestine. Additionally, in mouse models, the transmission of opportunistic pathogens, like Klebsiella, from the oral cavity to the intestine has been shown to induce inflammation [30]. The oral cavity presents a potential reservoir for a wide range of opportunistic pathogens that have been linked to GI disorders.

\section{Potential relationship between oxygen and disruptor taxa} Several colonic GI disorders are linked to increased oxygen levels in the lumen resulting from decreased epithelial integrity and inflammation [49]. However, the barrier properties of the small intestine, an absorptive organ, are different from those of the colon. To our knowledge, shifts in absolute abundance of microbes capable of aerobic respiration and anaerobes have not been quantitatively studied previously in the human small intestine. The highly correlated abundance of both strict and facultative anaerobes that we observed could be a function of the oxygen gradients in the gut from the epithelial surface to the center of the lumen [50]. In our study, when diversity collapsed and disruptor taxa bloomed, the microbial composition shifted away from strict anaerobes to taxa capable of aerobic respiration. One clear outlier was a Clostridium classified as C.perfringens, which is a strict anaerobe but was highly correlated with the Enterobacteriaceae genera classified as disruptors. Previous mutualistic relationships between aerobic and anaerobic species that could help facilitate colonization have been observed in other studies with Bacteroides fragilis and either Klebsiella pneumonia or Escherichia coli $[51,52]$. We have previously hypothesized that the surprising coexistence of aerobe-anaerobe communities can occur in multi-stable systems, and that these communities can persist due to hysteresis [51]. Although multi-stability and hysteresis have not yet been documented in the gut microbiome, this phenomenon could explain the unexpected coexistence and persistence of aerobe-anaerobe communities in the small intestine.

\section{Disruptor taxa predict SIBO classification and likelihood of $\mathrm{Gl}$ symptoms}

Clinically, SIBO is classified by culture of duodenal aspirates on aerobic MacConkey agar or measurement of exhaled hydrogen and methane after intake of a fermentable sugar solution $[10,53]$. The main disruptor taxa (Enterobacteriaceae) grow well on MacConkey agar plates, which may explain the high correlation between SIBO classification and samples with disruptor taxa. It is commonly hypothesized that overgrowth of these taxa in the small intestine is responsible for the gas production detected during a breath test, and our study further supports this understanding because we found a correlation between bloating symptoms (attributable to gas production) and disruptor taxa. Future studies should determine whether individuals with and without high loads of disruptor taxa yield positive breath test results. Our findings support a strong relationship between overgrowth of specific disruptor taxa and GI symptoms in subjects with SIBO. High total microbial load alone in the small intestine was not associated with GI symptoms usually observed in subjects with SIBO and other GI conditions and diseases. Microbial culture is never perfect and will not capture all taxa associated with SIBO and GI conditions. However, our data suggest that SIBO diagnosis via microbial culture should focus on quantification of a specific group of disruptor taxa (Enterobacteriaceae) rather than total microbial load. Additionally, SIBO diagnosis via quantitative sequencing should focus on the absolute abundance of the seven disruptor taxa identified in this study.

Lactobacillus seemed to be an exception among the disruptor taxa in several ways. It commonly co-occurred with other disruptors; however, it was also present in many "normal" samples at low abundance. Additionally, when present at high total loads in the presence of other disruptor taxa, Lactobacillus load had a negative correlation with bloating score. However, Lactobacillus also dominated several samples that had no other disruptor taxa but had high symptom scores. It should also be noted that individuals taking probiotics $(N=49)$ did not have increased prevalence or abundance of Lactobacillus in the duodenum. Overall, finer taxonomic resolution may be required to decipher the role of different Lactobacillus species and strains. Their impact on human health is likely also dependent on the overall microbial community and host environment.

Although most patients in this study have various GI complications that could result in abdominal symptoms independent of a microbial component, patient samples with high loads of disruptor taxa had a substantially higher likelihood of having many severe GI symptoms. However, total microbial load alone did not associate with GI symptoms. Of the 13 cytokines and chemokines 
measured, only IL8 levels were significantly higher in the serum of patients with disruptor taxa, potentially indicating an associated local inflammatory process. Future studies that analyze biopsy transcriptomes would be needed to determine whether there is an associated host response, such as immune infiltration or epithelial stress responses in regions with disruptor taxa and/or high total microbial loads.

We initiated this study with four expectations, only one of which was supported by our data. Because mice are coprophagic and humans are not, we expected to see a dramatic difference between mouse and human smallintestine microbiomes. We indeed observed large quantitative and qualitative differences between the two. However, we were more surprised and educated by the three expectations that were shown to be incorrect. First, we expected microbial load in the human duodenum to have a bimodal distribution, with low microbial loads for non-SIBO patients and much higher load for SIBO patients, which our findings did not support (Fig. 5B). Second, because stomach acid and bile acid secretions isolate the duodenum from the upper GI tract and because the unidirectional flow of digesta and the ileocecal valve isolate the small intestine from the colon, we expected to find a unique population of microbes in the duodenum. We were surprised by the extent to which the oral microbiota appeared to influence the smallintestine microbiota (Figs. 1C and 2). Third, we expected to see microbiomes dominated by taxa generally thought of as commensals like Lactobacillus and Bifidobacterium. We were surprised by the prevalence and abundance of taxa known to be human pathogens (Figs. 1C and $3 \mathrm{~B}$ ), especially given that the small intestine is an immune-rich, absorptive organ with a loose mucus structure that likely permits substantial exposure to microbial cells and microbial-associated proinflammatory molecules.

\section{Limitations}

An acknowledged limitation of the study is that there are no healthy controls. All participants had some GI condition warranting the EGD procedure, which could bias our dataset and mask our ability to perceive relationships between microbial abundances and patient symptoms. New sampling techniques may be required to reduce the procedural risk involved with sampling healthy controls. Additionally, all collected samples in this study were from the lumenal contents of the duodenum. Distal regions of the small intestine may reveal further insights, and mucosal biopsies could be more indicative of mucosa-associated microbes that interact closely with the host. Although short amplicon sequencing allowed for more samples to be included in this study, utilizing shotgun sequencing approaches to reveal species- and strain-level resolution could provide additional insights, especially with regard to disruptor taxa and potential transmission of taxa from saliva to the duodenum. Additionally, DNA-based analyses can only inform which microbes are in a sample, not whether they are actively performing a function. RNA-based analyses, either $16 \mathrm{~S}$ rRNA or meta-transcriptomics, may shed additional light on which microbes are resident vs transient members of the duodenum and what functions they are performing. Finally, to truly unravel the connection between oral-to-small intestine microbial transmission and small-intestine microbe-host interactions, a more extensive characterization of the host is needed. Specifically, studies are needed to establish how variations in stomach acid levels, bile secretions, and GI motility impact the abundance and composition of smallintestine microbiota and in turn how the abundance and composition of small-intestine microbiota impacts immune and epithelial cell responses.

\section{Conclusions}

This study, with its acknowledged limitations, provides the largest dataset of the absolute abundance of microbiota from the human duodenum to date. We show a clear relationship between the human oral microbiota and that of the duodenum. Furthermore, absolute taxon abundances in the duodenum reveal a distinct subset of disruptor taxa, associated with human pathogens, that appear to displace common strict anaerobes. These same disruptor taxa are enriched in some individuals classified with SIBO and the absolute abundance of these disruptor taxa were associated with more severe GI symptoms. Future studies are needed to establish the host factors that control total microbial load in the duodenum, the mechanism of appearance and persistence of disruptor taxa, and how these disruptor taxa interact with the host.

\section{Methods}

Study population and design

The REIMAGINE (Revealing the Entire Intestinal Microbiota and its Associations with the Genetic, Immunologic, and Neuroendocrine Ecosystem) study was conceived to explore the relationships between the small-intestine microbial populations and different conditions and diseases [3]. Male and female subjects aged 18-80 years undergoing standard-of-care upper endoscopy (esophagogastroduodenoscopy, EGD) without colon preparation were prospectively recruited. All subjects were required to fast (from both solids and liquids, including water) starting at midnight the night before the procedure. The study protocol was approved by the Institutional Review Board (IRB) at Cedars-Sinai Medical Center, and subjects provided written informed consent 
prior to participation (IRB Protocol: 00035192). Data presented here represents a retrospective analysis of this prospectively collected information.

\section{Questionnaires}

Prior to EGD, all subjects completed a study questionnaire documenting demographic information and family and medical history, including GI disease and bowel symptoms, medication use, use of alcohol and recreational drugs, travel history, and dietary habits and changes. Subjects also reported any known underlying conditions, such as GI diseases and disorders, neurologic disease, hematologic disease, autoimmune disease, kidney disease, heart disease, and cancer. All medical information provided by subjects was verified through audits of medical records. All data were de-identified prior to analysis.

\section{Blood collection and analysis}

After completing the study questionnaire, fasting blood samples were collected in BD Vacutainer SST tubes (Becton Dickson, Franklin Lakes, NJ, USA). Levels of circulating pro- and anti-inflammatory cytokines and chemokines were analyzed on a Luminex FlexMap 3D (Luminex Corp., Austin, TX, USA) using a bead-based multiplex panel that included: GM-CSF, IFN $\gamma$, IL10, IL12P70, IL13, IL1B, IL2, IL4, IL5, IL6, IL8, MCP1, and TNF $\alpha$ (EMD Millipore Corp., Billerica, MA, USA, cat. \#HCYTOMAG-60K).

\section{Saliva and small-intestine lumenal sample collection}

Prior to EGD procedure, saliva was collected in a sterile $5 \mathrm{~mL}$ tube. During the EGD procedure, samples of duodenal lumenal fluid were procured using a customdesigned sterile aspiration double-lumen catheter (Hobbs Medical, Inc.) [28]. Duodenal aspirates (DA) were collected using a custom-designed sterile inner catheter which was pushed through a sterile bone wax cap only after the endoscopist entered the second portion of the duodenum, in order to reduce contamination from the mouth, esophagus, and stomach. After collection, samples were immediately placed on ice and transferred to the laboratory for further analysis.

\section{Aspirate processing and microbial culture}

Prior to microbial culture, an equal volume of sterile 6.5 $\mathrm{mM}$ dithiothreitol (DTT) prepared with RNase and DNase PCR-grade sterile water was added at a 1:1 ratio to each saliva and duodenal aspirate $(\sim 1 \mathrm{~mL})$ and the samples were vortexed until fully liquified $(\sim 30 \mathrm{~s})$ as described previously [28]. A 100- $\mu$ l aliquot of each duodenal sample (DA + DTT) was then serially diluted with $900 \mu \mathrm{L}$ sterile $1 \times$ PBS and plated on MacConkey agar (Becton Dickinson), and on blood agar (Becton
Dickinson). Plates were incubated at $37^{\circ} \mathrm{C}$ for $16-18 \mathrm{~h}$ under aerobic (MacConkey) or anaerobic (blood agar) conditions. Plates without bacterial growth after $18 \mathrm{~h}$ were re-incubated for an additional $18 \mathrm{~h}$. Colony forming units (CFU) were then counted electronically using a Scan 500 (Interscience, Paris, France). Saliva + DTT and the remainder of each DA+DTT were centrifuged at maximum speed (> 13,000 RPM) for $5 \mathrm{~min}$. The supernatant was removed, and $1 \mathrm{~mL}$ of sterile Allprotect reagent (Qiagen, Hilden, Germany) was added to the microbial pellet and then stored at $-80{ }^{\circ} \mathrm{C}$.

\section{DNA isolation}

On the day of the DNA isolation, DA pellets were thawed on ice and processed as described previously [28]. Microbial DNA was isolated using the MagAttract PowerSoil DNA KF Kit (Qiagen) on a KingFisher Duo (Thermo Fisher Scientific, Waltham, MA, USA), and quantified using Qubit dsDNA high sensitivity and Qubit dsDNA BR Assay kits (Invitrogen by Thermo Fisher Scientific) on a Qubit 4 Fluorometer (Invitrogen, Carlsbad, CA, USA).

\section{$16 \mathrm{~S}$ rRNA gene sequencing}

Extracted DNA was amplified, barcoded, and sequenced as described previously $[8,9,29]$. Briefly, amplification of the variable 4 (V4) region of the 16S rRNA gene was performed in $20 \mu \mathrm{L}$ duplicate reactions with: $8 \mu \mathrm{L}$ of 2.5× 5Prime Hotstart Mastermix (VWR, Radnor, PA, USA), $1 \mu \mathrm{L}$ of $20 \times$ Evagreen (VWR), $2 \mu \mathrm{L}$ each of $5 \mu \mathrm{M}$ forward and reverse primers (519F, barcoded 806R, IDT, CoralVille, IA, USA), $3.5 \mu \mathrm{L}$ of water, and $3.5 \mu \mathrm{L}$ of extracted DNA template. A CFX96 RT-PCR machine (BioRad Laboratories, Hercules, CA, USA) was used to monitor amplification reactions and all samples were removed in late exponential phase ( 10,000 FRU) to minimize chimera formation and non-specific amplification $[9,54,55]$. Amplification was performed under the following cycling conditions: $94{ }^{\circ} \mathrm{C}$ for $3 \mathrm{~min}$, up to 50 cycles of $94{ }^{\circ} \mathrm{C}$ for $45 \mathrm{~s}, 54{ }^{\circ} \mathrm{C}$ for $60 \mathrm{~s}$, and $72{ }^{\circ} \mathrm{C}$ for 90 s. Several samples were rerun after diluting the template as they showed non-exponential amplification in the undiluted sample, a sign of PCR inhibition. Amplified duplicates were pooled together and quantified with KAPA library quantification kit (Roche, Basel, Switzerland) and then all samples were pooled at equimolar concentrations with up to 96 samples per library. AMPureXP beads (Beckman Coulter, Brea, CA, USA) were used to clean up and concentrate libraries before final library quantification with a High Sensitivity D1000 Tapestation Chip (Agilent, Santa Clara, CA, USA). Illumina MiSeq sequencing was performed with a $2 \times 300$ bp reagent kit by Fulgent Genetics (Temple City, CA, USA). 
Raw reads were demultiplexed by Fulgent Genetics. Demultiplexed forward and reverse reads were processed with QIIME 22020.2 [56]. Loading of sequence data was performed with the demux plugin followed by quality filtering and denoising with the dada2 plugin [57]. Dada2 trimming parameters were set to the base pair where the average quality score dropped below thirty. All samples were rarefied to the lowest read depth present in all samples (45,386 reads) to decrease biases from varying sequencing depth between samples [58]. The q2-featureclassifier [59] was then used to assign taxonomy to amplicon sequence variants (ASV) with the Silva [60] 132 99\% OTUs references. Resulting read count tables were used for downstream analyses in IPython notebooks (see "Data availability" section).

\section{Klebsiella-specific qPCR}

Primers specific for the Klebsiella gltA gene [61] (F: 5' CAGGCCGAATATGACGAATTC-3'， R: 5'-CGGGTG ATCTGCTCATGAA-3') were first informatically evaluated for coverage across Klebsiella pneumoniae, Klebsiella oxytoca, and Klebsiella aerogenes via Primer-BLAST [62]. This primer set was found to have a perfect match against strains from all three tested Klebsiella species. These primers were also evaluated in the lab for specificity against Escherichia coli. No amplification after 40 cycles was observed with a DNA equivalent of $\sim 10^{6} \mathrm{E}$. coli cells from the Zymo microbial community DNA standard (Zymo Research, Irvine, CA, USA). Klebsiella qPCR was performed in $10 \mu \mathrm{L}$ reactions with $5 \mu \mathrm{L}$ of Ssofast Evagreen Supermix (Bio-Rad Laboraties), $0.5 \mu \mathrm{L}$ of $10 \mu \mathrm{M}$ gltA primers, and $3.5 \mu \mathrm{L}$ of water. A CFX96 RT-PCR machine (Bio-Rad Laboratories) was used for amplification with the following cycling conditions: 95 ${ }^{\circ} \mathrm{C}$ for $3 \mathrm{~min}, 40$ cycles of $95{ }^{\circ} \mathrm{C}$ for $15 \mathrm{~s}, 62{ }^{\circ} \mathrm{C}$ for $30 \mathrm{~s}$, and $68{ }^{\circ} \mathrm{C}$ for $30 \mathrm{~s}$. Estimated conversion of cycle threshold $(\mathrm{Cq})$ to copies $/ \mu \mathrm{L}$ was performed where a Cq of 22.4 equals 1000 copies/uL. Klebsiella load was then calculated by adjusting for dilutions and normalizing to the collected sample volume.

\section{Absolute abundance}

The total microbial load (bacteria and archaea) of each sample and the absolute abundance of each taxon in individual samples was determined as described previously $[9,29]$. Briefly, the Bio-Rad QX200 droplet dPCR system (Bio-Rad Laboratories) was utilized to measure the $16 \mathrm{~S}$ concentration in each sample with the following reaction components: 1X QX200 EvaGreen Supermix (Bio-Rad), $500 \mathrm{nM}$ forward primer, and $500 \mathrm{nM}$ reverse primer (519F, 806R) and thermocycling conditions: $95{ }^{\circ} \mathrm{C}$ for 5 min, 40 cycles of $95{ }^{\circ} \mathrm{C}$ for $30 \mathrm{~s}, 52{ }^{\circ} \mathrm{C}$ for $30 \mathrm{~s}$, and 68 ${ }^{\circ} \mathrm{C}$ for $30 \mathrm{~s}$, followed by a dye stabilization step of $4{ }^{\circ} \mathrm{C}$ for $5 \mathrm{~min}$ and $90{ }^{\circ} \mathrm{C}$ for $5 \mathrm{~min}$. The final concentration of $16 \mathrm{~S}$ rRNA gene copies in each sample was corrected for dilutions and normalized to the extracted sample volume.

For each sample, the input-volume-normalized total microbial load from dPCR was multiplied by each amplicon sequence variant's (ASV) relative abundance to determine the absolute abundance of each ASV. No correlation between collected sample volume and measured bacterial load was observed. The average of all sample volumes for a specific sample type was used for a few samples (11 duodenum, 10 saliva) that were missing the starting volume information. A 95\% confidence interval of input volumes for duodenum samples ranged from 0.18 to $1.93 \mathrm{~mL}$ indicating that the estimated input volume measurement would likely be up to $4 \times$ off in either direction while the total microbial load ranged 40,000X. Similarly, a 95\% confidence interval of input volumes for saliva samples ranged from 0.36 to $1.28 \mathrm{~mL}$ indicating that the estimated input volume measurement would likely be up to $2 \times$ off in either direction while the total microbial load ranged $82 \mathrm{X}$.

\section{Poisson quality filtering}

Two separate quality-filtering steps based on Poisson statistics were used to determine the statistical confidence in the measured values. First, a 95\% confidence interval was calculated from the repeated measures of water blanks. Samples with a total microbial load below the upper bound of this confidence interval were removed from further analysis.

Second, the limit of detection (LOD) in terms of relative abundance was determined for each sample. Sequencing can be divided into two separate Poisson sampling steps. First, an aliquot of sample is taken from the extracted sample and input into the library amplification reaction. The LOD of the library amplification step was determined by multiplying the total microbial load from $\mathrm{dPCR}$ by the input volume into the library amplification reaction and then finding the relative abundance corresponding to an input of three copies. Poisson statistics tells us that the likelihood of sampling one or more copies with an average input of three copies is $95 \%$. The second Poisson sampling step in sequencing arises from the number of reads generated from the amplified library. The accuracy of the second Poisson sampling step was previously shown [9] to follow a negative exponential curve, LOD $=7.115 *$ read depth $^{-0.115}$, between the total read depth and relative abundance at which $95 \%$ confidence of detection is observed. The minimum of the two described LODs (first determined per sample by total load, and second by sequencing depth) was then determined for each sample. For each sample, the abundance of any ASV with a relative 
abundance below the LOD was set to zero. After filtering, data tables for each taxonomic level were generated.

\section{Data transforms and dimensionality reduction}

For PCA, all absolute taxon abundances were logtransformed. To handle zeros, a pseudo-count of 0.1 reads was added to all taxon relative abundances before multiplying by each sample's total microbial load as determined by digital PCR. PCA was performed with the sklearn.decomposition.PCA function in Python. Ranked feature loadings for each taxon on a given principal component were determined by scaling the corresponding eigenvector by the maximum transformed value for that principal component axis.

\section{Statistical analysis and correlations}

Group comparisons (e.g., SIBO vs. no SIBO, saliva vs. duodenum) were analyzed using the non-parametric Kruskal-Wallis rank sums tests with Benjamini-Hochberg multiple hypothesis testing correction using SciPy.stats Kruskal function and statsmodels.stats.multitest multipletests function with the fdr_bh option.

Correlation coefficients were either Spearman or Pearson and corresponding $P$ values for all correlations were determined with scipy.stats.spearmanr or scipy.stats.pearsonr functions. Multiple hypothesis testing was performed for each group of correlations (e.g., taxa cocorrelations, cytokine correlations) separately using the Benjamini-Hochberg procedure.

\section{Supplementary Information}

The online version contains supplementary material available at https://doi. org/10.1186/s40168-021-01162-2.

Additional file 1: Figure S1. Total microbial load breakdown by age (A) and gender (B). Figure S2. Distribution of total microbial load from subpopulations of patients: taking probiotics $(\mathrm{N}=49)$, active smokers $(\mathrm{N}=$ 16), taking antibiotics in the past 6 months $(\mathrm{N}=100)$, or taking proton pump inhibitors (PPI, N=106). Figure S3. (A) Scatterplot comparing aerobic culture load from MacConkey plates to total load from $16 \mathrm{~S}$ quantitative sequencing of only the subset of bacteria that are known to grow on MacConkey plates (Escherichia-Shigella, Enterobacteriaceae, Enterococcus, and Aeromonas) ${ }^{1}$. (B) Scatterplot comparing anaerobic culture load, from blood agar plates, to total load from sequencing of prevalent bacteria that are expected to grow on blood agar plates (Prevotella, Streptococcus, Fusobacterium, Escherichia-Shigella) ${ }^{2}$. Red dashed line indicates limit of detection of quantitative sequencing method. $\mathrm{N}=$ 244. (Six patients in the study were lacking culture data). Figure S4. (A) Cycle threshold $(\mathrm{Cq})$ values yielded by qPCR with Klebsiella-specific primers. Duodenum aspirate samples were classified via quantitative sequencing as containing Enterobacteriaceae ("Entero +", $\mathrm{N}=22$ ) or not containing Enterobacteriaceae ("Entero -", $N=8$ ). (B) Total loads of Enterobacteriaceae (copies $/ \mathrm{mL}$ ) in duodenum aspirates as a factor of the approximate Klebsiella load (copies $/ \mathrm{mL}$ ). Enterobacteriaceae measurements are calculated based on 165 rRNA gene copies (8 copies/genome) and Klebsiella measurements are calculated based on the citrate synthase gene (gltA, 1 copy/genome). Figure S5. Receiver operating characteristic (ROC) curve using absolute loads of seven disruptor taxa (Enterobacteriaceae, Escherichia-Shigella, Clostridium sensu stricto 1 , Enterococcus, Romboutsia, Aeromonas, Bacteroides) identified in the sequencing data for SIBO classification. SIBO classification was made based on gold-standard aerobic culture results, $\geq 10^{3} \mathrm{CFU} / \mathrm{mL}$ of duodenal aspirate. Data points are connected by a line between each consecutive point. Figure S6. IL8 levels in samples with and without a Clostridium which, based on the V4 region of the $16 \mathrm{~S}$ rRNA gene, was classified as C. perfringens. Figure S7. Relationship between Lactobacillus load and bloating symptoms in samples containing additional (nonLactobacillus) disruptor taxa. Figure S8. Violin plots with data points overlaid for patient-reported symptom scores. Binary threshold for determining whether severe symptoms exist was set at the median score reported of each symptom, shown by the red-dashed lines. Figure S9. Disruptor taxa load separated by patient age: 18-39 ( $N=40), 40-49(\mathrm{~N}=31)$, 50-59 ( $N=58), 60-69(N=67), 70-83(N=54)$. Figure S10. Relationship between absolute abundance (greater than $10^{5}$ copies $/ \mathrm{mL}$ ) and relative abundance of disruptor loads (Spearman, $P=0.09$, not significant). Figure S11. Comparison of total microbial load between human duodenum, mouse duodenum, and mouse duodenum where the mice had coprophagy prevented via tail cup. Mouse data from Bogatyrev et al. $2020^{3}$. Reported $P$-values are from Kruskal-Wallis test. Table S1. Summary statistics for the patient cohort used in this study. All patients are from the REIMAGINE study ${ }^{4}$. Table S2. P-values from significance tests (Kruskal-Wallis) comparing total microbial load between selected subgroups of individuals. Significance is indicated with an asterisk. Table S3. Comparison between prevalence and relative abundance of all taxa in paired saliva and duodenum samples ( $N=21$ participants). Table S4. Two groups of taxa (light blue and dark blue) that have stronger co-correlations with another taxon than with total load. Significance values for all correlations and co-correlations were $P<0.001$

Additional file 2

\section{Acknowledgements}

We thank the Caltech Bioinformatics Resource Center for assistance with statistical analyses, Jenny Ji for related analyses and Natasha Shelby for contributions to writing and editing this manuscript. We acknowledge OpenMoji for use of the saliva and stool graphics in Fig. 1. We thank Stacy Weitsman, Walter Morales and Maria Jesus Villanueva-Milan from MAST for assistance with sample processing and data curation from the REIMAGINE study. We also thank the Gastroenterology team at Cedars-Sinai Medical Center for assistance with patient recruitment and endoscopy procedures.

\section{Authors' contributions}

Conceptualization, J.T.B., G.L., R.M., M.P., and R.F.I.. Methodology, J.T.B., G.L., S.C., R.S., C.C.. Formal analysis, J.T.B.. Investigation, J.T.B, G.L., and A.E.R. Resources, G.L. Data curation, J.T.B., and G.L. Writing-original draft, J.T.B. Writing-review and editing, J.T.B., G.L., A.R., R.M., M.P., and R.F.I. Visualization, J.T.B. Supervision, M.P., and R.F.I. The author(s) read and approved the final manuscript.

\section{Funding}

This work was supported in part by the Kenneth Rainin Foundation (20181207), the Jacobs Institute for Molecular Engineering for Medicine, and a National Institutes of Health Biotechnology Leadership Pre-doctoral Training Program (BLP) fellowship from Caltech's Donna and Benjamin M. Rosen Bioengineering Center (T32GM112592, to J.T.B.). The funders had no role in the design of the study, the collection, analysis, and interpretation of data, nor in writing the manuscript.

\section{Availability of data and materials}

Sequencing data generated during this study are available in the National Center for Biotechnology Information Sequence Read Archive repository under study accession number PRJNA674353. Raw data for each figure and IPython notebooks for data processing and figure generation are available through CaltechDATA: https://data.caltech.edu/records/1701.

\section{Declarations}

Ethics approval and consent to participate

The study was reviewed and approved by the Cedars-Sinai Medical Center IRB (Protocol \#00035192). All participants provided written informed consent prior to participation. 


\section{Consent for publication}

Not applicable.

\section{Competing interests}

The quantitative sequencing technology described in this publication is the subject of a patent application filed by Caltech. R.F.I. receives patent royalties from Bio-Rad related to droplet digital PCR

\section{Author details}

'Division of Biology and Biological Engineering, California Institute of Technology, 1200 E. California Blvd, Pasadena, CA 91125, USA. ${ }^{2}$ Medically Associated Science and Technology (MAST) Program, Cedars-Sinai Medical Center, Los Angeles, CA 90048, USA. ${ }^{3}$ Division of Chemistry and Chemical Engineering, California Institute of Technology, 1200 E. California Blvd, Pasadena, CA 91125, USA. ${ }^{4}$ Division of Digestive and Liver Diseases, Cedars-Sinai Medical Center, Los Angeles, CA 90048, USA. ${ }^{5}$ Division of Endocrinology, Diabetes, and Metabolism, Cedars-Sinai Medical Center, Los Angeles, CA 90048, USA.

Received: 15 June 2021 Accepted: 14 September 2021

Published online: 02 November 2021

\section{References}

1. Donaldson GP, Lee SM, Mazmanian SK. Gut biogeography of the bacterial microbiota. Nat Rev Microbiol. 2016;14(1):20-32. https://doi.org/10.1038/ nrmicro3552.

2. Yasuda K, Oh K, Ren B, Tickle TL, Franzosa EA, Wachtman LM, et al. Biogeography of the intestinal mucosal and lumenal microbiome in the rhesus macaque. Cell Host Microbe. 2015;17(3):385-91. https://doi.org/10.1 016/..chom.2015.01.015.

3. Leite GGS, Weitsman S, Parodi G, Celly S, Sedighi R, Sanchez M, et al Mapping the segmental microbiomes in the human small bowel in comparison with stool: a REIMAGINE study. Dig Dis Sci. 2020;65(9):2595-604. https://doi.org/10.1007/s10620-020-06173-x.

4. Johansson MEV, Sjövall H, Hansson GC. The gastrointestinal mucus system in health and disease. Nat Rev Gastroenterol Hepatol. 2013;10(6):352-61. https://doi.org/10.1038/nrgastro.2013.35.

5. Ko H-J, Chang S-Y. Regulation of intestinal immune system by dendritic cells. Immune Netw. 2015;15(1):1-8. https://doi.org/10.4110/in.2015.15.1.1.

6. Rios D, Wood MB, Li J, Chassaing B, Gewirtz AT, Williams IR. Antigen sampling by intestinal $M$ cells is the principal pathway initiating mucosal IgA production to commensal enteric bacteria. Mucosal Immunol. 2016;9(4): 907-16. https://doi.org/10.1038/mi.2015.121

7. Ebino KY. Studies on coprophagy in experimental animals. Jikken Dobutsu. 1993:42:1-9

8. Bogatyrev SR, Rolando JC, Ismagilov RF. Self-reinoculation with fecal flora changes microbiota density and composition leading to an altered bile-acid profile in the mouse small intestine. Microbiome. 2020;8(1):19. https://doi. org/10.1186/s40168-020-0785-4

9. Barlow JT, Bogatyrev SR, Ismagilov RF. A quantitative sequencing framework for absolute abundance measurements of mucosal and lumenal microbial communities. Nat Commun. 2020;11(1):2590. https://doi.org/10.1038/s41467020-16224-6.

10. Pimentel M, Saad RJ, Long MD, Rao SSC. ACG Clinical Guideline: Small Intestinal Bacterial Overgrowth. Official J Am Coll Gastroenterol | ACG. 2020;115:165-78.

11. Lupascu A, et al. Hydrogen glucose breath test to detect small intestinal bacterial overgrowth: a prevalence case-control study in irritable bowel syndrome. Aliment Pharmacol Ther. 2005;22(11-12):1157-60. https://doi. org/10.1111/j.1365-2036.2005.02690x.

12. Shah A, et al. Small intestinal bacterial overgrowth in irritable bowel syndrome: a systematic review and meta-analysis of case-control studies. Official J Am College Gastroenterol | ACG. 2020;115:190-201.

13. Roland $B C$, et al. Small intestinal transit time is delayed in small intestinal bacterial overgrowth. J Clin Gastroenterol. 2015:49:571-76.

14. Roland BC, Lee D, Miller LS, Vegesna A, Yolken R, Severance E, et al. Obesity increases the risk of small intestinal bacterial overgrowth (SIBO). Neurogastroenterol Motil. 2018;30(3):e13199. https://doi.org/10.1111/ nmo.13199.

15. Su T, Lai S, Lee A, He X, Chen S. Meta-analysis: proton pump inhibitors moderately increase the risk of small intestinal bacterial overgrowth. $J$ Gastroenterol. 2018;53(1):27-36. https://doi.org/10.1007/s00535-017-1371-9.
16. Quigley EMM, Murray JA, Pimentel M. AGA clinical practice update on small intestinal bacterial overgrowth: expert review. Gastroenterology. 2020;159(4): 1526-32. https://doi.org/10.1053/j.gastro.2020.06.090.

17. Pimentel M, Chang C, Chua KS, Mirocha J, DiBaise J, Rao S, et al. Antibiotic treatment of constipation-predominant irritable bowel syndrome. Dig Dis Sci. 2014;59(6):1278-85. https://doi.org/10.1007/s10620-014-3157-8.

18. Leite G, Morales W, Weitsman S, Celly S, Parodi G, Mathur R, et al. The duodenal microbiome is altered in small intestinal bacterial overgrowth. PLoS One. 2020;15(7):e0234906. https://doi.org/10.1371/journal.pone.0234906.

19. Jonsson $\mathrm{H}$. Segmented filamentous bacteria in human ileostomy samples after high-fiber intake. FEMS Microbiol Lett. 2013;342(1):24-9. https://doi. org/10.1111/1574-6968.12103.

20. Zoetendal EG, Raes J, van den Bogert B, Arumugam M, Booijink CCGM, Troost FJ, et al. The human small intestinal microbiota is driven by rapid uptake and conversion of simple carbohydrates. ISME J. 2012;6(7):1415-26. https://doi.org/10.1038/ismej.2011.212.

21. Hartman AL, Lough DM, Barupal DK, Fiehn O, Fishbein T, Zasloff M, et al, Human gut microbiome adopts an alternative state following small bowel transplantation. Proc Natl Acad Sci U S A. 2009;106(40):17187-92. https://doi. org/10.1073/pnas.0904847106.

22. Chen Y, Ji F, Guo J, Shi D, Fang D, Li L. Dysbiosis of small intestinal microbiota in liver cirrhosis and its association with etiology. Sci Rep. 2016; 6(1):34055. https://doi.org/10.1038/srep34055.

23. Saffouri GB, Shields-Cutler RR, Chen J, Yang Y, Lekatz HR, Hale VL, et al. Small intestinal microbial dysbiosis underlies symptoms associated with functional gastrointestinal disorders. Nat Commun. 2019;10(1):2012. https:// doi.org/10.1038/s41467-019-09964-7.

24. Zmora N, et al. Personalized Gut Mucosal Colonization Resistance to Empiric Probiotics Is Associated with Unique Host and Microbiome Features. Cell. 2018:174:1388-405.

25. Chen RY, Kung VL, Das S, Hossain MS, Hibberd MC, Guruge J, et al. Duodenal microbiota in stunted undernourished children with enteropathy. NewEngl J Med. 2020;383(4):321-33. https://doi.org/10.1056/NEJMoa1916004.

26. Knight R, Vrbanac A, Taylor BC, Aksenov A, Callewaert C, Debelius J, et al. Best practices for analysing microbiomes. Nat Rev Microbiol. 2018;16(7):41022. https://doi.org/10.1038/s41579-018-0029-9.

27. Morton J, Marotz C, Washburne A, Silverman J, Zaramela LS, Edlund A, et al. Establishing microbial composition measurement standards with reference frames. Nature Commun. 2019;10(1):2719. https:/doi.org/10.1038/541467-019-10656-5.

28. Leite GGS, Morales W, Weitsman S, Celly S, Parodi G, Mathur R, et al. Optimizing microbiome sequencing for small intestinal aspirates: validation of novel techniques through the REIMAGINE study. BMC Microbiol. 2019; 19(1):239. https://doi.org/10.1186/s12866-019-1617-1.

29. Bogatyrev SR, Ismagilov RF. Quantitative microbiome profiling in lumenal and tissue samples with broad coverage and dynamic range via a singlestep $16 \mathrm{~S}$ rRNA gene DNA copy quantification and amplicon barcoding. bioRxiv. 2020. https://doi.org/10.1101/2020.1101.1122.914705v914701.

30. Atarashi K, Suda W, Luo C, Kawaguchi T, Motoo I, Narushima S, et al. Ectopic colonization of oral bacteria in the intestine drives TH1 cell induction and inflammation. Science. 2017;358(6361):359-65. https://doi.org/10.1126/ science.aan4526.

31. Schmidt TSB, Hayward MR, Coelho LP, Li SS, Costea PI, Voigt AY, et al. Extensive transmission of microbes along the gastrointestinal tract. eLife. 2019;8:e42693. https://doi.org/10.7554/eLife.42693.

32. Ghasemi A, Zahediasl S. Normality tests for statistical analysis: a guide for non-statisticians. Int J Endocrinol Metab. 2012;10(2):486-9. https://doi.org/10. 5812/ijem.3505

33. Lagkouvardos I, Overmann J, Clavel T. Cultured microbes represent a substantial fraction of the human and mouse gut microbiota. Gut Microbes. 2017;8(5):493-503. https://doi.org/10.1080/19490976.2017.1320468.

34. Ma R, Sapwell N, Chung HKL, Lee H, Mahendran V, Leong RW, et al. Investigation of the effects of $\mathrm{pH}$ and bile on the growth of oral Campylobacter concisus strains isolated from patients with inflammatory bowel disease and controls. J Med Microbiol. 2015;64(4):438-45. https://doi. org/10.1099/jmm.0.000013

35. Marcotte H, Lavoie MC. Oral microbial ecology and the role of salivary immunoglobulin A. Microbiol Mol Biol Rev. 1998;62(1):71-109. https://doi. org/10.1128/MMBR.62.1.71-109.1998.

36. Hojo K, Nagaoka S, Ohshima T, Maeda N. Bacterial Interactions in Dental Biofilm Development. J Dent Res. 2009;88(11):982-90. https://doi.org/10.11 $77 / 0022034509346811$ 
37. Hopkins EGD, Roumeliotis TI, Mullineaux-Sanders C, Choudhary JS, Frankel G. Intestinal Epithelial Cells and the Microbiome Undergo Swift Reprogramming at the Inception of Colonic Citrobacter rodentium Infection. mBio. 2019;10(2): e00062-19. https://doi.org/10.1128/mBio.00062-19.

38. Argüello H, Estellé J, Zaldívar-López S, Jiménez-Marín Á, Carvajal A, LópezBascón MA, et al. Early Salmonella Typhimurium infection in pigs disrupts Microbiome composition and functionality principally at the ileum mucosa. Sci Rep. 2018;8(1):7788. https://doi.org/10.1038/s41598-018-26083-3.

39. Contijoch EJ, Britton GJ, Yang C, Mogno I, Li Z, Ng R, et al. Gut microbiota density influences host physiology and is shaped by host and microbial factors. eLife. 2019;8:e40553. https://doi.org/10.7554/eLife.40553.

40. Nguyen TLA, Vieira-Silva S, Liston A, Raes J. How informative is the mouse for human gut microbiota research? Disease Models Mechanisms. 2015;8(1): 1-16. https://doi.org/10.1242/dmm.017400.

41. Caruso V, Song X, Asquith M, Karstens L. Performance of Microbiome Sequence Inference Methods in Environments with Varying Biomass. mSystems. 2019;4(1):e00163-18. https://doi.org/10.1128/mSystems.00163-18.

42. Glassing A, Dowd SE, Galandiuk S, Davis B, Chiodini RJ. Inherent bacterial DNA contamination of extraction and sequencing reagents may affect interpretation of microbiota in low bacterial biomass samples. Gut Pathog. 2016;8(1):24. https://doi.org/10.1186/s13099-016-0103-7.

43. Gevers D, Kugathasan S, Denson LA, Vázquez-Baeza Y, van Treuren W, Ren $B$, et al. The treatment-naive microbiome in new-onset Crohn's disease. Cell Host Microbe. 2014;15(3):382-92. https://doi.org/10.1016/j.chom.2014.02.005.

44. Goldberg S, Cardash H, Browning H, Sahly H, Rosenberg M. Isolation of Enterobacteriaceae from the Mouth and Potential Association with Malodor. J Dent Res. 1997;76(11):1770-5. https://doi.org/10.1177/00220345970760110801.

45. Smith JL, Fratamico PM. In: Caballero B, Finglas PM, Toldrá F, editors. Encyclopedia of Food and Health. Oxford: Academic Press; 2016. p. 539-44.

46. Gonçalves MO, Coutinho-Filho WP, Pimenta FP, Pereira GA, Pereira JAA, Mattos-Guaraldi AL, et al. Periodontal disease as reservoir for multi-resistant and hydrolytic enterobacterial species. Lett Appl Microbiol. 2007;44(5):48894. https://doi.org/10.1111/j.1472-765X.2007.02111.x.

47. Sharara SL, Tayyar R, Kanafani ZA, Kanj SS. HACEK endocarditis: a review. Expert Rev Anti Infect Ther. 2016;14(6):539-45. https://doi.org/10.1080/14 787210.2016 .1184085$.

48. Karched M, Bhardwaj RG, Asikainen SE. Coaggregation and biofilm growth of Granulicatella spp. with Fusobacterium nucleatum and Aggregatibacter actinomycetemcomitans. BMC Microbiol. 2015;15:114.

49. Rigottier-Gois L. Dysbiosis in inflammatory bowel diseases: the oxygen hypothesis. ISME J. 2013;7(7):1256-61. https://doi.org/10.1038/ismej.2013.80.

50. Litvak Y, et al. Commensal Enterobacteriaceae Protect against Salmonella Colonization through Oxygen Competition. Cell Host Microbe. 2019;25:128-39.

51. Khazaei T, et al. Metabolic multi-stability and hysteresis in a model aerobeanaerobe microbiome community. Sci Adv. 2020;6(33):eaba0353.

52. Dejea CM, Fathi P, Craig JM, Boleij A, Taddese R, Geis AL, et al. Patients with familial adenomatous polyposis harbor colonic biofilms containing tumorigenic bacteria. Science. 2018;359(6375):592-7. https://doi.org/10.112 6/science.aah3648.

53. Rezaie A, Buresi M, Lembo A, Lin H, McCallum R, Rao S, et al. Hydrogen and Methane-Based Breath Testing in Gastrointestinal Disorders: The North American Consensus. Am J Gastroenterol. 2017;112(5):775-84. https://doi. org/10.1038/ajg.2017.46.

54. Acinas SG, Sarma-Rupavtarm R, Klepac-Ceraj V, Polz MF. PCR-induced sequence artifacts and bias: insights from comparison of two 165 rRNA clone libraries constructed from the same sample. Appl Environ Microbiol. 2005;71(12):8966-9. https://doi.org/10.1128/AEM.71.12.8966-8969.2005.

55. Suzuki MT, Giovannoni SJ. Bias caused by template annealing in the amplification of mixtures of 165 rRNA genes by PCR. Appl Environ Microbiol. 1996;62(2):625-30. https://doi.org/10.1128/aem.62.2.625-630.1996.

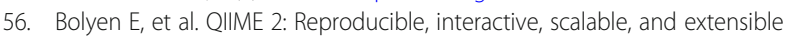
microbiome data science. Peer J. 2018;6:e27295v27292.

57. Callahan BJ, McMurdie PJ, Rosen MJ, Han AW, Johnson AJA, Holmes SP. DADA2: High-resolution sample inference from Illumina amplicon data. Nat Methods. 2016;13(7):581-3. https://doi.org/10.1038/nmeth.3869.

58. Weiss S, Xu ZZ, Peddada S, Amir A, Bittinger K, Gonzalez A, et al. Normalization and microbial differential abundance strategies depend upon data characteristics. Microbiome. 2017;5(1):27. https://doi.org/10.1186/s401 68-017-0237-y.

59. Bokulich NA, Kaehler BD, Rideout JR, Dillon M, Bolyen E, Knight R, et al. Optimizing taxonomic classification of marker-gene amplicon sequences with QIIME 2's q2-feature-classifier plugin. Microbiome. 2018;6(1):90. https:// doi.org/10.1186/s40168-018-0470-z.

60. Quast C, Pruesse E, Yilmaz P, Gerken J, Schweer T, Yarza P, et al. The SILVA ribosomal RNA gene database project: improved data processing and webbased tools. Nucleic Acids Res. 2013;41(Database issue):D590-6. https://doi. org/10.1093/nar/gks1219.

61. Clifford RJ, Milillo M, Prestwood J, Quintero R, Zurawski DV, Kwak YI, et al. Detection of Bacterial 165 rRNA and Identification of Four Clinically Important Bacteria by Real-Time PCR. PLoS One. 2012;7(11):e48558. https:// doi.org/10.1371/journal.pone.0048558.

62. Ye J, Coulouris G, Zaretskaya I, Cutcutache I, Rozen S, Madden TL. PrimerBLAST: A tool to design target-specific primers for polymerase chain reaction. BMC Bioinformatics. 2012;13(1):134. https://doi.org/10.1186/1471-21 05-13-134.

\section{Publisher's Note}

Springer Nature remains neutral with regard to jurisdictional claims in published maps and institutional affiliations.
Ready to submit your research? Choose BMC and benefit from:

- fast, convenient online submission

- thorough peer review by experienced researchers in your field

- rapid publication on acceptance

- support for research data, including large and complex data types

- gold Open Access which fosters wider collaboration and increased citations

- maximum visibility for your research: over $100 \mathrm{M}$ website views per year

At BMC, research is always in progress.

Learn more biomedcentral.com/submissions 